\title{
Simultaneous High Fe Charge State Measurements by Solar Energetic Particle and Solar Wind Instruments
}

\author{
M. A. Popecki ${ }^{1}$, T. H. Zurbuchen ${ }^{2}$, R. M. Skoug ${ }^{3}$, C. W. Smith ${ }^{4}$, A. B. Galvin ${ }^{1}$, \\ M. Lee', E. Möbius ${ }^{1}$, A. T. Bogdanov ${ }^{5}$, G. Gloeckler ${ }^{2}$, S. Hefti ${ }^{2}$, L. M. Kistler ${ }^{1}$, B. \\ Klecker $^{5}$, N. A. Schwadron ${ }^{2}$ \\ (1) Space Science Center and Department of Physics, University of New Hampshire, Durham, NH, USA; (2) The \\ University of Michigan Space Research Laboratory, Ann Arbor, MI, USA; (3) Los Alamos National Laboratory, \\ Los Alamos, NM, USA; (4) University of Delaware, Bartol Research Institute, Newark, DE, USA; (5) Max- \\ Planck-Institut für extraterrestrische Physik, Garching, Germany
}

\begin{abstract}
During the May 2-3, 1998 CME event, iron charge state distributions were observed on the ACE spacecraft simultaneously in both solar energetic particles and the solar wind. Surprisingly, common signatures were found, even though the energy of these two particle populations differ by about two orders of magnitude. At the beginning of the event a substantial shift towards higher charge states $(Q \geq 14)$ was detected for Fe nearly simultaneously in solar energetic particles by SEPICA, and in the solar wind by SWICS. The onset in the solar wind was somewhat more gradual, beginning approximately two hours before the rather sudden onset in the solar energetic particles. The high iron charge states coincided approximately with the magnetic cloud associated with the CME. Later, during the cloud passage, periods of high and unusually low, iron ionization states were observed in the solar wind. Just after the passage of the cloud, iron with high charge states again appeared in both energetic particles and the solar wind. Transport and acceleration scenarios will be discussed as possible explanations of this unusual event.
\end{abstract}

\section{INTRODUCTION}

With the launch of the Advanced Composition Explorer (ACE), it is possible for the first time to measure ionic charge states for the solar wind and solar energetic particles (SEPs) simultaneously. Charge states provide information about the source temperature, the SEP acceleration mechanisms and the propagation of SEPs through matter. Consequently, they can help identify the source population from which the SEPs are accelerated and can clarify details of the acceleration process.

In SEP events associated with a CME-driven interplanetary shock, known as gradual events (Reames (14)), the charge states of the SEPs are roughly similar to those in the solar wind (Klecker et al. (6), Gloeckler et al. (3)). The shock operates on ions in the corona or in the solar wind, where collisional effects are minimal during and after acceleration. In contrast, impulsive SEP events have been associated with flares (Reames
(14)). Typically these have ion charge states much higher than the solar wind (Möbius et al. $(11,12)$ ), suggesting that they originate from a hotter source region than the gradual SEPs.

The May 2-3, 1998 magnetic cloud contained solar wind and SEP ions that had first high, then low, iron charge states. The presence of these ions in the cloud along with the plasma and magnetic field data provide insights into the structure of a cloud and the acceleration processes responsible for the SEPs, and additional ionization may play an important role.

\section{OBSERVATIONS}

The early May 1998 period was very disturbed. Four large CMEs occurred between April 27 and May 6. The May 2-3 1998 solar wind observations in this paper were associated with a CME that lifted off the 
Sun on April 29, 1998. The details of the solar wind plasma parameters were presented by Skoug et al. (16). Using the SWEPAM and MAG instruments on ACE, they described a CME containing a cloud on May 2-3, 1998. They found an extended period of $\mathrm{He}+$ enhancement during and after the cloud. X-ray flares with optical counterparts were observed on the solar disk. None appeared to be well-connected to the Earth.

Figure 1 shows, from top to bottom, the proton speed from SWEPAM, the solar wind iron charge state, the SEP iron charge state and the SEP $\mathrm{Fe} / \mathrm{O}$ ratio at $0.47 \mathrm{MeV} /$ nuc. This energy is approximately the same at which the SEP charge states are measured. Further details of the solar wind mass and charge state composition for this event can be found in Gloeckler et al. (3). Also noted in Figure 1 are the boundaries of the CME and cloud, as well as observations of bidirectional electrons and two shocks. One shock occurred at 2123 on May 1, before the CME arrived, and the other at 17 UT on May 3, just after the cloud passed. The CME itself is considered to have begun at 3-5 UT on May 2, and the cloud extended from 13 UT on May 2 to 12UT on May 3. The measurements discussed here were made inside the cloud, and then just outside.

Before the cloud arrived, a gradual event was observed at ACE. The iron charge states were compatible with solar wind iron (Gloeckler et al. (3)), with $\mathrm{Q}_{\mathrm{Fe}}=9+, 10+$ and $11+$, as well as a tail toward higher charge states (Klecker et al. (7)). The first shock reached ACE during this event. The $\mathrm{Fe} / \mathrm{O}$ ratio in the SEPs was low at this time, which is an indicator of a gradual event (Reames (14)). Very little SEP Fe was observed between this event and the cloud.

When the cloud arrived at $\mathrm{ACE}$, a high charge component appeared in the solar wind and the SEPs at nearly the same time. The solar wind Fe, measured by SWICS, was mostly at $\mathrm{Q}_{\mathrm{Fe}}=16+$, with some even higher, but not shown because of a data processing artifact. SEP Fe, as observed by SEPICA, had a broad distribution centered around $\mathrm{QFe}_{\mathrm{Fe}}=15+$, and extending from $10+$ to $20+$. These values were significantly higher than during the preceding gradual event on May 1. Although the charge states in both the solar wind and SEPs increased simultaneously, it is important to note that the SEP charge distribution was not an exact replication of the solar wind. The $\mathrm{Fe} / \mathrm{O}$ ratio was also elevated, near one or two, in the SEP $\mathrm{Fe}$ at the beginning of the cloud. This was particularly so during the most intense SEP period of 13-14 UT.

At 21UT on day 122 (May 2, 1998), the charge state of iron decreased in the SEPs and more substantially in the solar wind. High and low iron charge states were present in the solar wind simultaneously, from 18 to 24 UT. A fresh injection occurred in the SEPs at 22 UT, as discerned from intensity vs. time curves (not shown). At the same time, an even lower charge iron population was measured in the solar wind $\left(\mathrm{Q}_{\mathrm{Fe}}=4\right.$ to $\left.5+\right)$. The SEP charge state distribution in this low charge period extended down to $\mathrm{Q}=6+$, and a $\mathrm{Q}=10-11+$ component persisted until 16 UT on May 3. This drop in charge state occurred at approximately the same time as a break in the bidirectional electrons. The $\mathrm{Fe} / \mathrm{O}$ ratio was variable in this period of mixed moderately high and very low SEP charge states, from as high as 1.3 to as low as 0.06 .

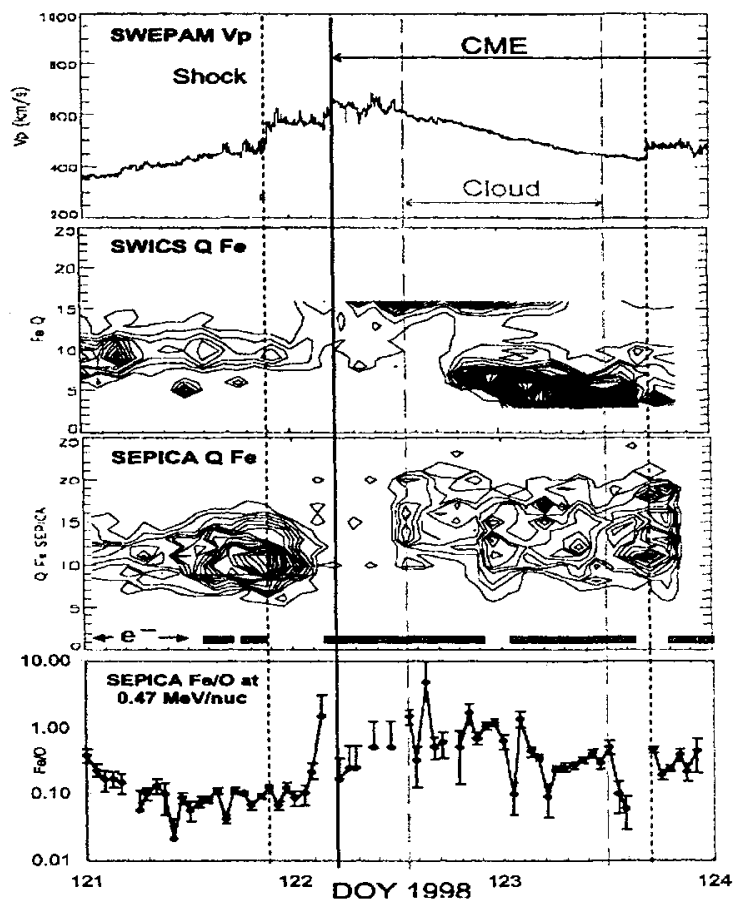

FIGURE 1. Solar wind proton speed, solar wind iron charge state, SEP iron charge state and SEP Fe/O are shown for the May 2-3, 1998 cloud CME period. Bidirectional electrons are indicated by horizontal bars in the SEPICA Q $\mathrm{Fe}$ panel.

Again, at 16 UT on day 123 , just after the cloud, a high charge component appeared in both the solar wind and SEPs, at the time of a shock passage. Iron $16+$ is prominent in the solar wind, and a strong enhancement at $\mathrm{Q}_{\mathrm{Fe}}=18-19+$ occurred in the SEPs. This enhancement occurred during a break in the bidirectional electrons.

\section{DISCUSSION}

The charge state information suggests the presence of three separate iron populations, in three separate flux tubes, within and then just outside the cloud. The first 
population had a sudden onset of high charge state iron in both SEPs and the solar wind at the beginning of the cloud. These were accompanied by bidirectional electrons and a high $\mathrm{Fe} / \mathrm{O}$ ratio in the SEPs. This simultaneous appearance of the SEPS and solar wind suggests that both were confined to a single flux tube. The high charge states, bidirectional electrons and the high $\mathrm{Fe} / \mathrm{O}$ ratio are consistent with an active, heated source region at the Sun. Bidirectional electrons can indicate the presence of field lines that have both footpoints at the Sun, with at least one near an active region (Kahler \& Reames (5), Gosling et al. (4)). Multiple flux tubes within a cloud have been discussed by Osherovich et al. (13), using the June 10-13 1993 Ulysses cloud observations. They inferred the cloud boundaries from solar wind proton density and the presence of ion acoustic waves.

The travel time for the solar wind is three to four days from the Sun to ACE, while it is only 6-7 hours for the SEP Fe. Therefore, their simultaneous appearance is not easy to understand in terms of propagation from a single event at the Sun. However, if a footpoint of the cloud remained magnetically connected to an active region on the Sun for several days, the following scenario is possible. The high charge solar wind could have left the Sun on this flux tube 3-4 days ago, while the high charge SEP Fe could have left from the same active region 6-7 hours ago, from a more recent eruption.

Previous observations have indicated that the footpoints of magnetic clouds may remain attached to the Sun long enough for a cloud to reach 1 AU. Mazur et al. (9) found SEP populations within magnetic clouds that had composition consistent with impulsive events. Kahler and Reames (5) observed proton SEPS that commenced during periods of bidirectional proton flows. Richardson and Cane (15) observed energetic particles in ejecta and concluded that their propagation was consistent with propagation along nonspiral, including looped, field lines. Larson, et al. (8) calculated field line lengths, using electron and Type III radio measurements for impulsive events that occurred while WIND was inside a cloud. They derived distances of 1.2 to $3 \mathrm{AU}$ for propagation through the center and along the outside boundary of the clouds respectively. The May 2, 1998, SEP iron would take 6-16 hours to travel such distances, assuming a pitch angle of zero degrees. Six X-ray flares of at least class $\mathrm{C}$ occurred during this time, including two at E34-35 and two at W04-05 longitude. Three of these flares were in active region 8210 , which was also active 3-4 days earlier.

Farrugia et al. (2) analyzed a magnetic cloud during which a rapid enhancement of $0.5-4 \mathrm{MeV} 1 \leq Z \leq 2$ ions occurred. The presence of the cloud was established in part by bidirectional ion flows. Locally shock accelerated ions that passed the spacecraft prior to the cloud became less intense near the cloud, suggesting that they could not efficiently enter. Farrugia et al. (2) concluded that the $0.5-4 \mathrm{MeV}$ ions were accelerated by a flare at the Sun a few hours earlier. Their access to the cloud suggested that the field lines remained connected to the Sun at least until the cloud passed the Earth.

We now turn to the second population of iron observed by SEPICA and SWICS in the May 2-3, 1998 period. The second population commenced nearly simultaneously in the solar wind and the SEPs. The charge states of iron were low in both the solar wind and SEPs. The solar wind iron contained lower charge states $\left(\mathrm{Q}_{\mathrm{Fe}} \geq 4+\right)$ than the SEPs. SEPICA could measure an SEP iron charge state as low as 2.2 in this event, but the lowest observed was $\mathrm{Q}_{\mathrm{Fe}}=6+$. The low limit of $\mathrm{Q}=2.2+$ is derived from the fact that SEPICA measures ionic charge via electrostatic deflection. A minimum deflection, and hence charge/energy ratio, is needed for complete measurement of the charge state distribution (Möbius et al. (10)).

It is conceivable that the low charge state SEP component was accelerated from the prominence material in the cloud by the shock that passed the spacecraft on May 3 at 17 UT. The $\mathrm{He}+$ in this material was described by Skoug et al. (16). The observed difference between the minimum charge states of the solar wind and SEPs may be interpreted as a consequence of the acceleration process. It should be noted, however, that the SEPs and the solar wind observed simultaneously in a flux tube are not truly contemporaries because of their different speeds. It is possible that the solar wind source that underwent acceleration had a charge state distribution similar to that of the SEPs.

Shock acceleration of heated solar wind in the cloud may be an alternative explanation for the high charge state SEP component at the beginning of the cloud. In that case, a recent eruption from the same active region would not be necessary.

The abrupt onset of the third population of high charge iron observed by SEPICA and SWICS, commencing with the break in the bidirectional electron flows, is again suggestive of a separate magnetic confinement of both solar wind and SEPs. This flux tube may be detached from the Sun at one of the two footpoints, however. Larson, et al. (9) noted breaks and dropouts in bidirectional electrons, without disruption to the local cloud magnetic field rotation. They concluded that those field lines were disconnected at one or both ends from the Sun. Armstrong et al. (1) observed a unidirectional ion beam in a flux rope structure. They interpreted this as hot coronal source, traveling along a field line whose 
antisunward end was connected to the outer heliosphere.

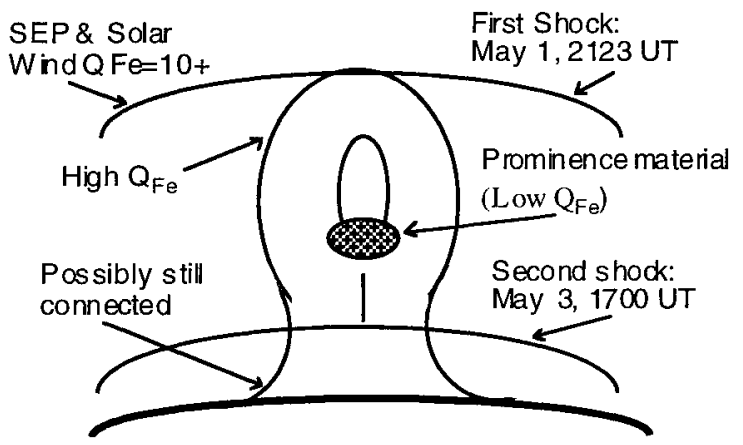

FIGURE 2. The May 2-3 1998 SEP and solar wind observations might be explained by a sequence in which a magnetic cloud passed the spacecraft, containing high charge state SEPs and solar wind on one flux tube. The SEPs either recently arrived from the same active region as the heated solar wind, or were shock accelerated from it. A second flux tube within the cloud, with lower charge state prominence material and SEPs derived from it, came next.

The third population of the solar wind and SEP ions occurred after the cloud was considered to have passed the spacecraft, possibly on a flux tube with footpoints separate from the cloud itself. This postcloud flux tube had a mixture of high and low charge state iron in it, so it was not strictly derived from an active region. The presence of flare-related SEPs through the end of a cloud was also noted by Mazur et al. (9). Wimmer-Schweingruber et al. (17) observed high charge states for solar wind iron in the material trailing the magnetic cloud in the November 7-8, 1997, active period.

These three distinct iron populations, as identified by their charge state and their $\mathrm{Fe} / \mathrm{O}$ ratio, and organized by breaks in the bidirectional electron flows, indicate that the spacecraft sampled at least three flux tubes within and then just outside a magnetic cloud. The high iron charge states in both solar wind and SEPs, just inside the cloud, are suggestive that the footpoints of the cloud were near an active region on the Sun for 3-4 days.

Finally, the possibility of shock acceleration on cloud field lines in the low charge state portion of the event may provide interesting insights on SEP acceleration from the solar wind. The acceleration of the SEPs, whether by an impulsive event or shock acceleration, could be better understood by including measurements of ${ }^{3} \mathrm{He}$ abundance and solar wind mass composition. The ${ }^{3} \mathrm{He}$ is an indicator of flare-associated (impulsive) acceleration. If it were observed in the initial portion of the cloud, where the iron charge states were high, then both the solar wind and SEP iron could be coming from active solar regions. However, if the mass composition of the solar wind was similar to that of the SEPs, then shock acceleration would be a plausible explanation.

\section{ACKNOWLEDGMENTS}

The authors are grateful to the many unnamed individuals at the University of New Hampshire, the Max-Planck-Institut fur extraterrestrische Physik and the Technical University of Braunschweig for their enthusiastic contributions to the completion of the ACE SEPICA instrument and the S3DPU. The work on the SEPICA instrument was supported by NASA under Contracts NAS5-32626 and NAG5-6912.

\section{REFERENCES}

1. Armstrong, T.P., et al., GRL, 21, 1747-1750, (1994).

2. Farrugia, C.J, et al., JGR, 98, 7621-7632, (1993).

3. Gloeckler, G., et al., GRL, 26, 157-160, (1999).

4. Gosling, J.T. J. Birn and M. Hesse, GRL, 22, 869-872, (1995).

5. Kahler, S.W. and D.V. Reames, JGR, 96, 9419-9424, (1991).

6. Klecker,B., et al., Proc. of the 26th Int'l Cosmic Ray Conf., eds. D. Kieda,et al., 1999, 6, pp.83-86.

7. Klecker, B., et al., ACE2000 Symp. Proc.s, 2000.

8. Larson, D.E., et al., GRL, 24, 1911-1914, (1997).

9. Mazur, J.E., et al., GRL, 25, 2521-2524, (1998).

10. Mobius, E., et a., Space Sci. Rev., 86, 449-495 (1998).

11. Moebius, E., et al., Proc. of the 26th Int'l Cosmic Ray Conf,, eds. D. Kieda,et al., 1999, 6, pp. 87-90.

12. Möbius, E., et al., ACE2000 Symp. Proc., 2000.

13. Osherovich V.A., et al., GRL, 26, 401-404, (1999).

14. Reames, D.V., Sp Sci Rev, 90 (3/4), $413-491$ (1999).

15. Richardson, I.G. and H.V. Cane, JGR, 101, 2752127532, (1996)

16. Skoug, R.M., et al., GRL, 26, 161-164, (1999).

17. Wimmer-Schweingruber, R.F., O. Kern and D.C. Hamilton, GRL, 26, 3541-3544, (1999). 